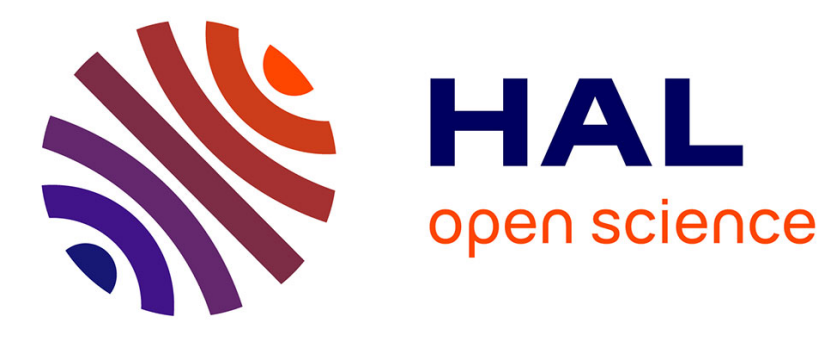

\title{
Contrôler rien, mais tous les jours
}

\author{
Alain Vanier
}

\section{To cite this version:}

Alain Vanier. Contrôler rien, mais tous les jours . Topique - Revue freudienne, 2008, Analyse quatrième, 2 (103), pp.49-57. 10.3917/top.103.0049 . hal-01521938

\section{HAL Id: hal-01521938 \\ https://hal.science/hal-01521938}

Submitted on 7 Jun 2017

HAL is a multi-disciplinary open access archive for the deposit and dissemination of scientific research documents, whether they are published or not. The documents may come from teaching and research institutions in France or abroad, or from public or private research centers.
L'archive ouverte pluridisciplinaire HAL, est destinée au dépôt et à la diffusion de documents scientifiques de niveau recherche, publiés ou non, émanant des établissements d'enseignement et de recherche français ou étrangers, des laboratoires publics ou privés. 


\section{Contrôler rien, mais tous les jours}

Alain Vanier

Si la crise ouverte au cœur de la psychanalyse - crise permanente et irréductible- est sa transmission, alors le contrôle est son symptôme.

En 1922, l'analyse didactique devient obligatoire, mais elle n'a pas besoin d'être aussi approfondie qu'une analyse thérapeutique. En 1927, sous l'influence de Ferenczi, on estime que les analystes doivent être plus analysés que leurs patients. Mais, quatre-vingts ans plus tard, le contraste entre l'abondance des travaux sur l'analyse didactique, sur l'analyse de l'analyste et la maigreur de la littérature sur le contrôle est saisissant ; à l'exception, sans doute, de celle produite par le $\mathrm{IV}^{\mathrm{e}}$ Groupe qui a fait de cette question le pivot de son nouage institutionnel. Ailleurs, fort peu de chose, si ce n'est ici ou là un texte très critique, polémique, manifestant une difficulté persistante inscrite dès le début de cette pratique et dont les différentes conceptions sont en tension depuis le temps de Vilma Kovàcs et de son texte fondamental. Il n'y a pas grand-chose à ajouter à ce texte, la plupart des problèmes que pose le contrôle y sont déjà indiqués. Ces tensions qui travaillent la conception et la pratique du contrôle sont toujours irrésolues aujourd'hui, surtout si l'on veut bien considérer nos pratiques.

Il y a près de quarante ans, Conrad Stein mettait en question le statut psychanalytique du contrôle dans la formation. Il distinguait «trois options », le contrôle pouvant être :

- Une pratique de surveillance du candidat et de sa pratique débutante,

- Une nouvelle psychanalyse car il est souvent une demande déguisée d'analyse,

- Ou encore l'enseignement d'une technique.

À ces trois options, on peut ajouter, avec Jean Clavreul, une pratique de défense de la respectabilité des institutions psychanalytiques. Ces différents points 
sont-ils jamais absents des pratiques du contrôle aujourd'hui comme hier? Les interroger déstabilise une certaine suffisance qui règle trop souvent les rapports du psychanalyste « reconnu » à son propre savoir. On pourra d'ailleurs interrogerla nécessité d'un contrôleur «reconnu» ou souvent qualifiéd'»expérimenté» dans cette pratique. Le savoir en jeu dans l'analyse est-il cumulatif? Si l'enjeu du contrôle est bien que l'analyste sache un peu ce qu'il fait, la question demeure, comme l'a formulée Nathalie Zaltzman : qu'attend-on du contrôle qui ne peut s'atteindre autrement ${ }^{1}$ ?

Le lien du contrôle à l'institution est à l'origine de l'analyse de contrôle : «Les stagiaires doivent à de courts intervalles faire un rapport au directeur de la policlinique », écrit Sandor Rado. Le directeur, puis d'autres, engagés dans cette pratique de contrôle, peuvent interrompre la cure si le candidat est incompétent ${ }^{2}$. Cette possibilité, tombée en désuétude semble-t-il, a ouvert la voie à une des dérives du contrôle, le contrôleur dirigeant la cure à travers le contrôlé, véritable exercice de ventriloquie analytique. Pour autant, on notera dans l'acte de fondation de l'École freudienne de Paris que «le contrôle s'impose [...] et d'abord pour protéger celui qui y vient en position de patient. » Or ce souci de protection de l'usager introduit au cœur de la formation quelque chose de contradictoire avecl'analyse.

Conrad Stein évoque, avec un courage qu'il faut souligner, son aventure personnelle du contrôle, dont l'impasse est le fait, selon lui, de la conjonction de trois hommes : le contrôleur, l'analyste et celui qui a adressé la patiente; trois hommes qui font institution, conduisant celle-ci au suicide ; la constitution d'un groupe se fait toujours sur un refoulement, si ce n'est sur un rejet. Il souligne pourtant l'utilité du contrôle, mais conteste son exigibilité à des fins de « reproduction de l'espèce analytique » selon le mot d'Eitingon.

Certes, en déplaçant le temps de l'articulation du contrôle à l'institution, quelque chose semble changer. Critiquant le dispositif d'avant-coup qui permet à l'institution d'autoriser la pratique analytique, c'est-à-dire un passage à l'acte de l'institution autorisant la pratique accompagnée d'un contrôle institué, Lacan constatait que « le psychanalyste ne s'autorise que de lui-même », à entendre comme un constat et non comme une licence généralisée. Par conséquent, c'est seulement dans l'après-coup que l'institution prend acte - ou non-d'une pratique qui est engagée sans qu'elle l'autorise, pas plus qu'elle ne décide du temps du contrôle. Or, le début de la pratique est souvent le fait d'un acting out, ce qui laisse une chance pour que cet acte soit travaillé analytiquement. Le contrôle peut-il être le lieu de ce travail ? Ce n'est pas sa fonction première, mais il n'est

1. N. Zaltzman, communication à la Journée du IVe Groupe O.P.L.F. « Contrôle, supervision, analyse quatrième », les 3 et 4 février 2007 à Paris, publiée dans ce numéro de Topique.

2 Rappelons que contrôle est formé de «contre» et de «rôle», et désignait un registre tenu en double, l'un servant à vérifier l'autre (d'où « contre »). Cf. A. Rey, Dictionnaire historique de la langue française, Paris, Le Robert, 1992. 
pas rare qu'il soit le lieu de cette élaboration. Mais de là se déduit la question du contrôle effectué avec son analyste, ouverte par les Hongrois, ou au moins la persistance d'une analyse personnelle pendant les premiers contrôles.

Pour revenir sur ce point, force est d'admettre qu'on ne s'en tire jamais à bon compte avec la question de l'institution. Elle rattrape celui qui la contourne, le choix des contrôleurs, leur poids dans les modalités de reconnaissance qui, si elle ne porte plus sur la pratique, garantit la formation à laquelle le contrôle contribue.

Le contrôle comme enseignement d'une technique est un autre versant du contrôle. Un livre comme celui de Greenson donne la mesure d'une telle option. Se pose alors la question de ce qu'il faut attendre de l'analyse personnelle si la méthode se transmet dans un strict compagnonnage. Le risque est celui d'une pratique qui ne produise que des standards.

Pourtant, qui peut dire qu'il n'a jamais donné de conseils, d'indications lors d'un contrôle? L'impossibilité de Freud d'écrire un traité sur la technique, la réfutation de ce terme par Lacan proposant celui d'éthique pour contrer la ritualisation de la pratique et situer différemment la position du psychanalyste, ont mis le terme de technique en disgrâce. Pourra-t-on absolument soutenir qu'il n'y a pas de technique analytique ? Celle-ci prend peut-être place au point paradoxal où est conduit l'analyste à la fin de sa propre analyse - là est peut-être la ressource du contrôle, si l'on veut bien entendre le terme de technique autrement que dans ses résonances contemporaines. Il y a sans doute une dimension de «métier», à critiquer au titre de «l'habitude», qui poussait Maud Mannoni à recommander des contrôles multiples avec des psychanalystes différents, ou Joyce Mc Dougall à proposer pour la formation du psychanalyste un «contrôle avec un lacanien très classique, un kleinien très classique, ainsi qu'avec quelques winnicottiens et un analyste d'enfant».

Une autre facette, avancée parVilma Kovàcs, metl'accent surle contre-transfert pour que «l'étudiant apprenne la façon correcte de le manier». Ce prolongement de la psychanalyse didactique doit amener à la conscience du candidat «ses tendances libidinales jusqu'ici refoulées, [le] familiariser avec la structure de son caractère». Car à la différence de l'approche éducative d'autres formations, «l'analyste, lui, doit toujours prendre connaissance de ses désirs enracinés dans ses instincts ». Dans cette perspective, le contrôle a lieu pendant l'analyse personnelle et doit être «mené de bout en bout par l'analyste didacticien ».

La radicalisation de cette approche ramène à l'analyse personnelle de l'analyste. Roger Perron le relève : à trop attirer l'attention sur le contre-transfert, on risque de transformer le contrôle en analyse. Jean-Luc Donnet souligne aussi les limites du discours sur le contre-transfert ${ }^{3}$. En effet, il peut conduire aussi

3. J.-L. Donnet, communication à la Journée du IV`Groupe O.P.L.F. « Contrôle, supervision, analyse quatrième », les 3 et 4 février 2007 à Paris, publiée dans ce numéro de Topique. 
bien à une nouvelle tranche d'analyse ou à une supervision. Cette hésitation ne souligne-t-elle pas une difficulté à distinguer contrôle et analyse ? Le contrôle serait-il la poursuite de l'analyse par d'autres moyens? Il est vrai que nombre de demandes de contrôle sont des demandes d'analyse et, dans certains cas, de première analyse. Mais réduire le contrôle à l'analyse personnelle laisse en plan la fonction du contrôle et sa spécificité, or tout le monde est d'accord sur sa nécessité, mais personne sur ses modalités. Moustapha Safouan proposera comme définition du contrôle «l'analyse des transferts et des contre-transferts de l'analyste contrôlé », au sens où analyser le transfert, c'est traiter le désir, le transfert étant à la fois la voie vers la reconnaissance du désir et en même temps ce qui y fait obstacle. Il n'est pas utile de revenir ici sur le contre-transfert, cette «notion assez approximative » selon François Perrier, si ce n'est pour rappeler qu'on ne peut nier le phénomène. Il désigne quelque chose d'essentiel qui concerne au plus haut point le contrôle, mais le terme même risque de rabattre la position de l'analyste sur une conception duelle de la situation analytique, d'individu à individu, beaucoup trop imaginaire. L'accent mis par le psychanalyste sur la surveillance de ses propres émois comme guide du traitement conduit parfois à ces exposés caricaturaux où le récit d'une cure se ramène au récit des éprouvés du psychanalyste sans qu'on puisse se faire la moindre idée du patient en question.

Il me semble que ces différentes facettes du travail de contrôle et les critiques correspondantes renvoient à la variété de ce qui se présente dans ce qu'on peut appeler la clinique du contrôle - qui, remarquons-le, reste à produire. Celleci est variable au plus haut point en fonction des analystes, des patients, etc. Il y a des demandes de contrôle qui sont des demandes d'analyse, et cet aspect peut-il être absent du contrôle car cette demande, la demande de contrôle, ne tend-elle pas à remettre en selle le sujet supposé savoir, ce qui en ferait aussi une procédure de résistance à l'analyse ? Certaines demandes sont liées au fait que la chute du sujet supposé savoir au principe du mouvement de la cure n'est pas liée au processus analytique mais à une difficulté dans l'analyse personnelle, celle-ci s'interrompant par résistance à cette disparition.

Il y a des demandes de contrôle qui sont des demandes d'autorisation où, par exemple, celui qui demande vient présenter successivement chaque situation pour obtenir répétitivement une permission d'engager la cure.

Il y a des demandes de contrôle qui s'adressent au savoir à partager face à la solitude, voire au désarroi dans lequel le jeune psychanalyste se trouve quand il découvre, dans le meilleur des cas, que son bagage théorique ne lui est pas d'un grand secours. Il attend alors d'un surcroît de technique le comblement du vide qu'il ressent dans la situation de transfert. Ainsi ce sentiment d'impuissance ou d'imposture que ressent le jeune analyste, et qui le conduit à faire appel au savoir comme remède pour combler ce vide, ne masque-t-il pas le fait que l'imposture est au cœur de la relation analytique, comme le rappelait Jean 
Clavreul ? Il ajoutait que le débutant le ressente n'est pas mal, car, effectivement, il est du ressort du transfert qu'on soit pris pour un autre. Il est, en effet, préférable qu'il ne se prenne pas pour l'Autre ou qu'il se croit analyste, d'autant que l'enjeu est moins l'addition d'un savoir que le repérage de la position qu'il tient dans le dispositif ${ }^{4}$. Ce que le psychanalyste sait, ce n'est pas ce qu'il est supposé savoir. Il a appris de son analyse ce qui le détermine, il s'est dépris de ses identifications et doit savoir ce qui l'oriente sur ce plan, et il doit encore se défaire de ce qui fait obstacle à l'apparition d'un savoir, de ce savoir d'un autre ordre qui est en jeu dans l'analyse. Clavreul soulignait qu'en ce sens, le contrôle n'est pas la transmission d'un savoir, mais plutôt la mise à jour de ce qui fait obstacle au désir de savoir et pousse l'analyste à poursuivre avec les patients l'expérience engagée par sa propre analyse. Ce qui y fait obstacle, ce sont les théories implicites de l'analyste - ses préjugés, disait Freud-, théories qu'il s'agit de faire chuter pour qu'un autre savoir advienne.

En somme, il s'agit de démoïser la position du psychanalyste. On remarquera qu'un texte de Lacan comme «La direction de la cure » se déploie entre une première affirmation - c'est l'analyste qui dirige la cure, au sens où il ne dirige pas le patient ni la vie de celui-ci, mais donne l'orientation du traitementet une formule finale - c'est le désir inconscient qui oriente la cure-désir de l'analyste, aurait-il pu dire plus tard, comme conjoignant ces deux propositions. Pour autant, iln'y a pas lieu de confondre le temps de la passe et celui du contrôle. Si la passe est le moment de ce désir, et sa procédure, le dispositif pour le cerner, le contrôle n'est pas contrôle de l'advenue du désir de l'analyste, il serait plutôt travail de la façon dont l'analyste y fait obstacle. La passe n'a pas supprimé le contrôle, condition nécessaire mais pas suffisante à régler le problème de la transmission.

De même, la question institutionnelle peut se ramener à celle de la communauté. Qu'est-ce que les psychanalystes ont en commun, si ce n'est l'exercice de ce lien social qu'est la psychanalyse qui isole pour chacun ce qui ne peut se mettre en commun ? Mais c'est aussi ce qui met au travail la communauté des analystes et le mouvement par lequel l'analyste soutient le désir de savoir qui l'anime. Conrad Stein, lui-même, contestant le contrôle et soutenant que les demandes de contrôle peuvent toutes se ramener à des demandes d'analyse, admettait qu'avec cette perspective on butait sur le mirage de l'analyse absolue, d'une analyse sans restes dont l'effet d'ouverture ne se refermerait pas. Or il y a un reste inéliminable qui peut être mis en relation avec le pointaveugle de l'analyse, avec l'objet au sens que Lacan donne à ce terme, ce fragment de réel que la symbolisation échoue à traiter totalement. C'est en ce lieu qu'une certaine dimension, savoir-faire et non technique, peut venir en jeu dans le

4. Car l'enjeu, pourra dire Lacan, n'est pas seulement dans l'affaire ce que l'analyste entend faire de son patient, mais aussi ce que l'analyste entend que son patient fasse de lui. 
contrôle, à la façon dont la construction pallie l'interprétation quand celle-ci devient impossible. Ainsi je soutiendrai assez volontiers, avec Maud Mannoni, qu'en matière de contrôle, il convient de «prendre ce qui vient». Sans s'obséder sur la pureté de la demande de contrôle et de considérer plutôt, comme dans les prémisses d'une analyse, que, là aussi, la charge nous revient de conduire le demandeur au contrôle analytique au prix des détours nombreux, parfois longs, mais incontournables qui sont ceux de toute pratique conséquente du contrôle, s'il est analytique. Ces figures évoquées, ces «options » au sens de Conrad Stein, me semblentêtre les voies des engluements imaginaires qui, malgré tout, conduisent au contrôle. Comme lors des préliminaires d'une cure, il s'agit - effet de l'acte-d'instituer la psychanalyse. Tout analyste n'a-t-il pas à refaire le chemin de Freud, à répéter son geste - l'abandon de l'hypnose-car c'est à ce titre que l'on s'adresse à nous. Demande adressée à un maître qui saurait. C'est en ce sens que je comprends la recommandation de Conrad Stein que nous devons être des «pionniers de la psychanalyse », formule qui rejoint le conseil de Freud d'aborder chaque cure comme s'il s'agissait de la première.

Si l'on considère ces options du contrôle comme les figures imaginaires de ce qu'est le contrôle analytique, alors celui-ci se dessine en creux. Pour cela je m'appuierai sur Lacan, sur l'une des rares définitions du contrôle, peutêtre la seule, qu'il ait donnée, très tôt, dès le début de son enseignement. Dans ce bref passage, il commence par une observation triviale : le contrôleur, comme personne tierce, entend souvent mieux que le contrôlé. En premier lieu, parce qu'il est soustrait à l'embarras imaginaire de la situation analytique, moins pris dans la dérive des identifications ; mais aussi parce que la situation de contrôle, situation de témoignage indirect, obéit aux lois ordinaires de la transmission : l'émetteur reçoit du récepteur son propre message sous une forme inversée. La place tierce du contrôleur fait donc qu'il entend mieux, et, dès lors, peut faire entendre. Le contrôleur dans le contrôle «manifeste une seconde vue $[\ldots]$ qui rend pour lui l'expérience au moins aussi instructive que pour le contrôlé ». Il ajoute : «La raison de cette énigme est que le contrôlé y joue le rôle de filtre, voire de réfracteur du discours du sujet, et qu'ainsi est présentée toute faite au contrôleur une stéréographie dégageant déjà les trois ou quatre registres où il peut lire la partition constituée par ce discours. » Plus loin : « Si le contrôlé pouvait être mis par le contrôleur dans une position subjective différente de celle qu'implique le terme sinistre de contrôle (avantageusement remplacé, mais seulement en langue anglaise, par celui de supervision), le meilleur fruit qu'il tirerait de cet exercice serait d'apprendre à se tenir luimême dans la position de subjectivité seconde où la situation met d'emblée le contrôleur. Il y trouverait la voie authentique pour atteindre ce que la classique formule de l'attention diffuse, voire distraite, de l'analyste n'exprime que très approximativement. » 
Lacan proposera plusieurs termes pour remplacer celui de contrôle, ainsi « super-audition », mais reprendra le terme initial d'»analyse de contrôle ». On notera d'ailleurs que le terme de contrôle s'est maintenu dans l'orientation lacanienne.

«Se tenir lui-même dans cette position de subjectivité seconde», que l'on pourrait traduire par division du sujet, position divisée de l'analyste, bien que cette lecture soit anachronique au regard de ce texte. Le rôle du contrôleur estil seulement de lire par-dessus l'épaule de l'analyste ? Notre pratique, le réel de notre clinique nous divise. Face à cela, il peut être tentant de quêter de nouvelles identifications comme, par exemple, la demande adressée à un analyste admiré.

Or, c'est moins comme sujet que nous intervenons dans le traitement qu'avec cette division même qui marque le désir, et met en jeu et aux commandes l'objet - objet en tant que manquant, même si c'est comme semblant qu'il est demandé, passage obligé. C'est cette place de l'objet offerte comme semblant, pour quoi l'analyste n'a pas lieu de se prendre, que Lacan met au principe du discours analytique. Objet de la division même de l'analyste comme échec à toute maîtrise quelle qu'elle soit. Cet objet est un principe d'altérité radicale. Il est ce qui garantit l'altérité même de l'Autre, que le névrosé se dévoue à faire exister et qui constitue le ressort même du transfert. L'objet $a$ est demandé sur le versant de l'objet partiel sur fond d'objet perdu, mais soutenu, fondé, par ce que la cure dénudera, l'objet comme réel, comme ce rien fondamentalement manquant, jamais perdu ${ }^{5}$.

Le psychanalyste est une place, un opérateur dans la cure. Cette place ne peut être tenue que par quelqu'un qui est passé par l'expérience d'une analyse. La place que l'analyste occupe, qu'il soutient dès la feinte initiale quand la demande lui est adressée, n'est possible qu'à ne pas s'égarer dans le dédale des identifications unifiantes où il est convié. Ainsi cette position de «subjectivité seconde » manifeste la division de l'analyste quand il opère. Entre les deux pôles de sa division, ce qu'il présente est le lieu où il écoute, ce qui est mis en jeu dans cette division est ce rien qu'est le réel même de l'objet manquant. C'est ce qui vient s'articuler, se déplier dans cette situation seconde - et non pas double, comme pourrait l'indiquer l'étymologie du mot contrôle-articulée à la mise en fonction de ce tiers dont le contrôleur occupe pour un temps la place. Le contrôleur incarne alors un des pôles de cette division ; le contrôle articule ainsi ce qui fait défaut dans le savoir pour que puisse se totaliser l'expérience, défaut où se manifeste ce «qu'il est possible de saisir en un éclair au-delà des limites du savoir». Le contrôleur occupe un temps cette place - contrôler avec «quelques autres »-pourqu'ensuite l'analyste puisse soutenir «l'expérience que nous

5. En ce sens, on peut soutenir que l'objet transitionnel de Winnicott a comme fonction de donner sens de perte au manque. 
avons à contrôler tous les jours », contrôler ce presque rien crucial, dont l'ajustement, la bonne place, est décisif pour la cure : contrôler rien mais tous les jours.

\section{Alain VANIER}

14 bis, rue Raynouard

75016 Paris

\section{BIBLIOGRAPHIE}

Clavreul J. (1984) «Interview sur le contrôle », Patio, n², Paris, Evel.

Colonomos F. (1985) On forme des psychanalystes. Paris : Denoël.

Greenson R.R., Technique et pratique de la psychanalyse, trad. F. Robert. Paris : P.U.F., 1977.

Kovacs V. (1936) « Analyse didactique et analyse de contrôle », trad. M. Moreau Ricaud, Topique, $\mathrm{n}^{\circ} 18$. Paris : Epi, 1977.

Lacan J. (1953) «Fonction et champ de la parole et du langage» Écrits. Paris : Seuil, 1966.

Mc Dougall J. (1984) «Interview sur le contrôle», Patio, n². Paris : Evel.

Perron R. (2002) « Contrôle (psychanalyse sous) », Dictionnaire international de la psychanalyse, dir. A. de Mijolla. Paris : Calmann-Lévy.

M. Safouan (1975) «La question des contrôles », Lettres de l'École freudienne, $\mathrm{n}^{\circ}$ 16, Paris.

Stein C. (1970) «Sur la pratique des cures contrôlées », La mort d'Édipe. Paris: DenoëlGonthier, 1977.

Topique $\mathrm{n}^{\circ} 1$, « La formation du psychanalyste » (P.U.F.), Topique $\mathrm{n}^{\circ} 18$, « Trajets analytiques » (Epi), Topique n 97 « L'Écoute transmise » (L’Esprit du Temps).

VanierA. (2006) «Mouvements del'objet», Mensuel del'École de Psychanalyse des Forums du Champ lacanien, $\mathrm{n}^{\circ} 18$.

\section{Alain Vanier - Contrôler rien, mais tous les jours}

Résumé : Si le contrôle est une pièce incontournable de la formation du psychanalyste, sa fonction reste problématique, et les travaux qui tentent de la fonder particulièrement peu nombreux au regard de ceux qui traitent de l'analyse de l'analyste ou de l'enseignement. Ce texte tente de repérer ce qui peut être spécifique dans cette pratique, à partir des critiques qui la mettent pertinemment en question sans pour autant la contester, puisqu'elle reste effective dans l'ensemble de la communauté analytique.

Mots-clés : Contrôle - Supervision - Formation du psychanalyste. 


\begin{abstract}
Alain Vanier - Controlling Nothing But Doing It Every Day
Summary : Supervision plays a central role in the training of analysts but its function remains a problematic question and research into this question is scarce in comparison with that done on the analysis of the analyst or teaching. This text aims at highlighting the specificities of supervision based on criteria that will enable us to question it without forasmuch refuting it, since supervision is practised across the whole analytical community.
\end{abstract}

Key-words : Control - Supervision - Training analysts. 